\title{
A NEW ANALYTICAL MODEL FOR AXI-SYMMETRIC CAVITIES
}

\section{D.Burrini, C.Pagani, L.Serafini, INFN-Milan \\ Via Celoria 16, 20133 Milano, Italy}

In this paper we derive a general off-axis expansion valid for axi-symmetrical em. fields. With the application of general arguments a new method to determine analytically off-axis field components is presented, starting with the knowledge of the on-axis distribution of the longitudinal field component. Analytical and numerical em. fields off-axis are compared, in the case of RF monopole modes in a cavity, showing very good agreement up to a radial off-axis position witch is comparable to the wavelength. The relevance of the method on applications in analytical study of particles dynamics is discussed and a calculation of the transverse momentum imparted by the field to a relativistic particle is reported: this is of interest for dark current, beam halo and RF injector analysis. Another kind of application in cavity design is mentioned.

\section{GENERAL EXPANSION}

In deriving a general off-axis expansion for axi-symmetric em. field, we consider both TE-like and TM-like waves. The cylindrical symmetry of the field allows us to write:

$$
\begin{aligned}
& \left(\begin{array}{l}
E_{z}^{T M}(r, z, t) \\
H_{z}^{T E}(r, z, t)
\end{array}\right)=A_{0}(z, t)+A_{2}(z, t) r^{2}+. .+A_{2 N}(z, t) r^{2 N} \\
& \left.\left(\begin{array}{l}
E_{r}^{T M}(r, z, t) \\
H_{r}^{T E}(r, z, t)
\end{array}\right)=B_{1}(z, t) r+B_{3}(z, t) r^{3}+. .+B_{2 N+1}(z, t) r^{2 N+1} 1\right) \\
& \left(\begin{array}{c}
E_{\varphi}^{T E}(r, z, t) \\
H_{\varphi}^{T M}(r, z, t)
\end{array}\right)=C_{1}(z, t) r+C_{3}(z, t) r^{3}+. .+C_{2 N+1}(z, t) r^{2 N+1}
\end{aligned}
$$

where $A_{0}(z, t)=\left(\begin{array}{l}E_{z}(r=0, z, t)-T M \\ H_{z}(r=0, z, t)-T E\end{array}\right)$ is the field on-axis. We want to obtain a formula which allows to write the field off-axis, by the knowledge of the field on-axis shown above.

To do this, the simplest way is to consider the following Maxwell equations in empty space $(\rho=0, J=0$, $\left.\varepsilon_{r}=\mu_{r}=1\right)$;

$\nabla \cdot \mathbf{E}=0 \quad ; \quad \nabla^{2} \mathbf{E}-\frac{1}{c^{2}} \frac{\partial^{2}}{\partial t^{2}} \mathbf{E}=0$

$\left\{\nabla \times\left(\begin{array}{c}\mathbf{E}^{T E} \\ \mathbf{H}^{T M}\end{array}\right)(r, z, t)\right\}_{z}=\left(\begin{array}{c}-\mu_{0} \\ \varepsilon_{0}\end{array}\right) \frac{\partial}{\partial t}\left(\begin{array}{c}H_{z}^{T E} \\ E_{z}^{T M}\end{array}\right)(r, z, t)$

Assuming an harmonic time dependence as $e^{i \omega t}$ we project in cylindrical co-ordinates and replace the expressions of the fields in eq.1, obtaining the following recurrence relations:

$$
\begin{aligned}
& 4 N^{2} A_{2 N}(z, t)=-\left\{\frac{\partial^{2}}{\partial z^{2}} A_{2(N-1)}(z, t)+k^{2} A_{2(N-1)}(z, t)\right\} \\
& 2(N+1) B_{2 N+1}(z, t)+\frac{\partial}{\partial z} A_{2 N}(z, t)=0 \\
& 2(N+1) C_{2 N+1}(z, t)=i k\left(\begin{array}{l}
Z_{0}^{-1} \\
-Z_{0}
\end{array}\right) A_{2 N}(z, t)
\end{aligned}
$$

where $Z_{0}=\sqrt{\mu_{0} / \varepsilon_{0}}$ is the vacuum impedance and $k=\omega / c$. The following treatment holds for resonant modes and/or for single Fourier components (angular frequency $\omega$ ) of a cylindrical wave: the time dependence left in the field coefficients $A_{i}, B_{i}, C_{i}$ is meant to represent a slowly varying envelope behaviour, under the hypothesis $\partial A_{i} / \partial t<<\omega A_{i}$.

Defining the unidimensional Helmoltz operator like $\Phi\left(A_{0}(z, t)\right)=\frac{\partial^{2}}{\partial z^{2}} A_{0}(z, t)+k^{2} A_{0}(z, t)$ we can write the coefficients $A_{2 N}(z, t)$ in a compact form as functions of the on-axis distribution field $A_{0}(z, t)$. This is written as $A_{2 N}(z, t)=\frac{(-1)^{N}}{2^{2 N} N !^{2}} \Phi^{N}\left(A_{0}(z, t)\right)$ where for $N=0$ we have $\Phi^{0}\left(A_{0}(z, t)\right)=A_{0}(z, t)$. Now with the recurrence relation obtained previously and the form of the em. field due to the symmetry, we finally have:

$$
\begin{aligned}
& \left(\begin{array}{c}
E_{z}^{T M} \\
H_{z}^{T E}
\end{array}\right)=\operatorname{Re}\left[\sum_{N=0}^{v} \frac{(-1)^{N}}{N !^{2}} \Phi^{N}\left(A_{0}(z, t)\right)\left(\frac{r}{2}\right)^{2 N}\right] \\
& \left.\left(\begin{array}{c}
E_{r}^{T M} \\
H_{r}^{T E}
\end{array}\right)=\operatorname{Re}\left[\sum_{N=0}^{v} \frac{(-1)^{N+1}}{N !^{2}(N+1)} \Phi^{N}\left(\frac{\partial}{\partial z} A_{0}(z, t)\right)\left(\frac{r}{2}\right)^{2 N+1}\right] 4\right) \\
& \left(\begin{array}{c}
H_{\varphi}^{T M} \\
E_{\varphi}^{T E}
\end{array}\right)=\operatorname{Re}\left[i k\left(\begin{array}{l}
Z_{0}^{-1} \\
-Z_{0}
\end{array}\right) \sum_{N=0}^{v} \frac{(-1)^{N}}{N !^{2}(N+1)} \Phi^{N}\left(A_{0}(z, t)\right)\left(\frac{r}{2}\right)^{2 N+1}\right]
\end{aligned}
$$

These are the most general expressions for off-axis expansion of any axis-symmetrical wave. Taking $k=0$ in the previous expressions, the Helmoltz operator becomes $\Phi\left(A_{0}(z)\right)=\frac{d^{2}}{d z^{2}} A_{0}(z)=A_{0}{ }^{I I}(z)$ and we get the case of electrostatic field for TM modes, and the magnetostatic field for TE modes:

$$
\begin{aligned}
& \left(\begin{array}{l}
E_{z}^{S T A} \\
H_{z}^{S T A}
\end{array}\right)=A_{0}-A_{0}^{I I}\left(\frac{r}{2}\right)^{2}+\frac{1}{4} A_{0}^{I V}\left(\frac{r}{2}\right)^{4}-\frac{1}{36} A_{0}^{V I}\left(\frac{r}{2}\right)^{6}+\ldots \\
& \left(\begin{array}{l}
E_{r}^{S T A} \\
H_{r}^{S T A}
\end{array}\right)=\frac{-r}{2} A_{0}^{I}+\frac{1}{2} A_{0}^{I I I}\left(\frac{r}{2}\right)^{3}-\frac{1}{12} A_{0}^{V}\left(\frac{r}{2}\right)^{5}+\frac{1}{128} A_{0}^{V I I}\left(\frac{r}{2}\right)^{7}+.
\end{aligned}
$$


The above expressions are written for a few terms of expansion and are in agreement with ref.[1]: they can be used to obtain off-axis fields in magnetostatic and/or electrostatic lenses.

\section{SPATIAL HARMONICS EXPANSION AND TM010- $\pi$ MODE}

In a multi-cell accelerating structure, we know that under certain conditions the field on-axis can be expressed in Floquet form, see [2], so $A_{0}=\operatorname{Re}\left[\sum_{n=-\infty}^{\infty} b_{n} \exp i\left(\omega t-k_{n} z\right)\right]$, with $k_{n}=\left(\frac{\psi+2 \pi n}{d}\right)$ while $\psi$ is the phase shift per cavity cell. Applying the Helmoltz operator to the Floquet form we obtain:

$$
\Phi^{N}\left(A_{0}(z, t)\right)=k^{2 N} \operatorname{Re}\left[\sum_{n=-\infty}^{+\infty} b_{n}\left(1-\kappa_{n}^{2}\right)^{N} \exp i\left(\omega t-k_{n} z\right)\right]
$$

where $\kappa_{n}=k_{n} / k$. Substituting in the expressions of the general expansion (eqs.4), we have:

$$
\begin{gathered}
\left(\begin{array}{c}
E_{z}^{T M} \\
H_{z}^{T E}
\end{array}\right)(r, z, t)=\operatorname{Re}\left[\sum_{n=-\infty}^{+\infty} b_{n} e^{i\left(\omega t-k_{n} z\right)} \sum_{N=0}^{v} \frac{\left(\kappa_{n}^{2}-1\right)^{N}}{N !^{2}}\left(\frac{k r}{2}\right)^{2 N}\right] \\
\left(\begin{array}{c}
E_{r}^{T M} \\
H_{r}^{T E}
\end{array}\right)(r, z, t)=\operatorname{Re}\left[-i \sum_{n=-\infty}^{+\infty} \kappa_{n} b_{n} e^{i\left(\omega t-k_{n} z\right)}\right. \\
\left.\sum_{N=0}^{v} \frac{\left(\kappa_{n}^{2}-1\right)^{N}}{N !^{2}(N+1)}\left(\frac{k r}{2}\right)^{2 N+1}\right] \\
\left(\begin{array}{c}
H_{\varphi}^{T M} \\
E_{\varphi}^{T E}
\end{array}\right)(r, z, t)=\operatorname{Re}\left[i\left(\begin{array}{c}
Z_{0}^{-1} \\
-Z_{0}
\end{array}\right) \sum_{n=-\infty}^{+\infty} b_{n} e^{i\left(\omega t-k_{n} z\right)}\right. \\
\left.\sum_{N=0}^{v} \frac{\left(\kappa_{n}^{2}-1\right)^{N}}{N !^{2}(N+1)}\left(\frac{k r}{2}\right)^{2 N+1}\right]
\end{gathered}
$$

This is the spatial harmonics expansion for a general axisymmetric em. field, which is described by the spatial harmonics coefficients $b_{n}$. Setting $b_{-(n+1)}=b_{n}{ }^{*}$ and $\psi=\pi$, we obtain the well-known $\mathrm{TM}_{010-} \pi$ standing mode used in many accelerating structures.

The accelerating field on-axis, $A_{0}(z, t)$, in this particular case becomes $A_{0}(z, t)=\operatorname{Re}\left[\left(\sum_{n-o d d} a_{n} \exp \left(\frac{i \pi n}{d} z\right)\right) \exp i\left(\omega t+\phi_{0}\right)\right]$, where $\phi_{0}$ is the initial phase of the wave and $a_{n}$ are the harmonics satisfying the relation above. Since the Helmoltz operator doesn't act on the time variables, we may omit the exponential factor for simplicity. Defining $k^{\prime}=\pi / d$ we apply it on this expression obtaining:
$E_{z}^{\pi}(z, r)=\operatorname{Re}\left[\sum_{n} a_{n} e^{i k^{\prime} n z} \sum_{N=0}^{v} \frac{\left(n^{2} k^{\prime 2}-k^{2}\right)^{N}}{N !^{2}}\left(\frac{r}{2}\right)^{2 N}\right]$

$\left.E_{r}^{\pi}(z, r)=\operatorname{Re}\left[-i \sum_{n} n a_{n} e^{i k^{\prime} n z} \sum_{N=0}^{v} \frac{k^{\prime}\left(n^{2} k^{\prime 2}-k^{2}\right)^{N}}{N !^{2}(N+1)}\left(\frac{r}{2}\right)^{2 N+1}\right] 5\right)$

$H_{\varphi}^{\pi}(z, r)=\operatorname{Re}\left[\frac{1}{Z_{0}} \sum_{n} a_{n} e^{i k^{\prime} n z} \sum_{N=0}^{v} \frac{k\left(n^{2} k^{2}-k^{2}\right)^{N}}{N !^{2}(N+1)}\left(\frac{r}{2}\right)^{2 N+1}\right]$

In multi-cell structures for relativistic particles we have $d=\lambda / 2$ so $k=k^{\prime}$, and the above expressions become simpler as follows:

$$
\begin{aligned}
& E_{z}^{\pi}(z, r)=\left\{\begin{array}{c}
\sum_{n} a_{n} \cos (n k z) \quad ; \quad r=0 \\
\sum_{n} a_{n} \cos (n k z) \sum_{N=0}^{v} \frac{\left(n^{2}-1\right)^{N}}{N !^{2}}\left(\frac{k r}{2}\right)^{2 N} ; r \neq 0
\end{array}\right. \\
& E_{r}^{\pi}(z, r)=\sum_{n} n a_{n} \sin (n k z) \sum_{N=0}^{v} \frac{\left(n^{2}-1\right)^{N}}{N !^{2}(N+1)}\left(\frac{k r}{2}\right)^{2 N+1} \\
& H_{\varphi}^{\pi}(z, r)=\frac{1}{Z_{0}} \sum_{n} a_{n} \cos (n k z) \sum_{N=0}^{v} \frac{\left(n^{2}-1\right)^{N}}{N !^{2}(N+1)}\left(\frac{k r}{2}\right)^{2 N+1}
\end{aligned}
$$

For $n=1$ and $N=0$ these expressions give the linear field expansion used in several beam dynamics studies, see [2]. If $v \rightarrow \infty$, the series in eqs.5 converge to modified Bessel functions of first kind, setting $\kappa=k^{\prime} / k$ we have:

$$
\begin{aligned}
& E_{z}^{\pi}(r, z)=\sum_{n} a_{n} \cos \left(n k^{\prime} z\right) I_{0}\left(k r \sqrt{(n \kappa)^{2}-1}\right) \\
& E_{r}^{\pi}(r, z)=\sum_{n} n a_{n} \sin \left(n k^{\prime} z\right) \frac{\kappa I_{1}\left(k r \sqrt{(n \kappa)^{2}-1}\right)}{\sqrt{(n \kappa)^{2}-1}} \\
& H_{\varphi}^{\pi}(r, z)=\frac{1}{Z_{0}} \sum_{n} a_{n} \cos \left(n k^{\prime} z\right) \frac{\kappa I_{1}\left(k r \sqrt{(n \kappa)^{2}-1}\right)}{\sqrt{(n \kappa)^{2}-1}}
\end{aligned}
$$

where the first harmonic term is obtained as limit for $n \rightarrow 1$; if $\kappa=1$ we obtain expressions in agreement with previous studies [2]. We compared the analytic field expression $r \cdot H_{\varphi}^{\pi}(r, z)$ (eqs.6) with the corresponding numerical values generated by SUPERFISH in the case of a typical superconducting multicell cavity whose boundary is shown in fig.2 (solid line). The range of application of the method is $k r / 2<1$, the maximum order of expansion is about $v \cong n$ for a chosen set of harmonics. The result of the comparison is shown in fig.1, for a set of nine harmonics and seventh-order expansion. 


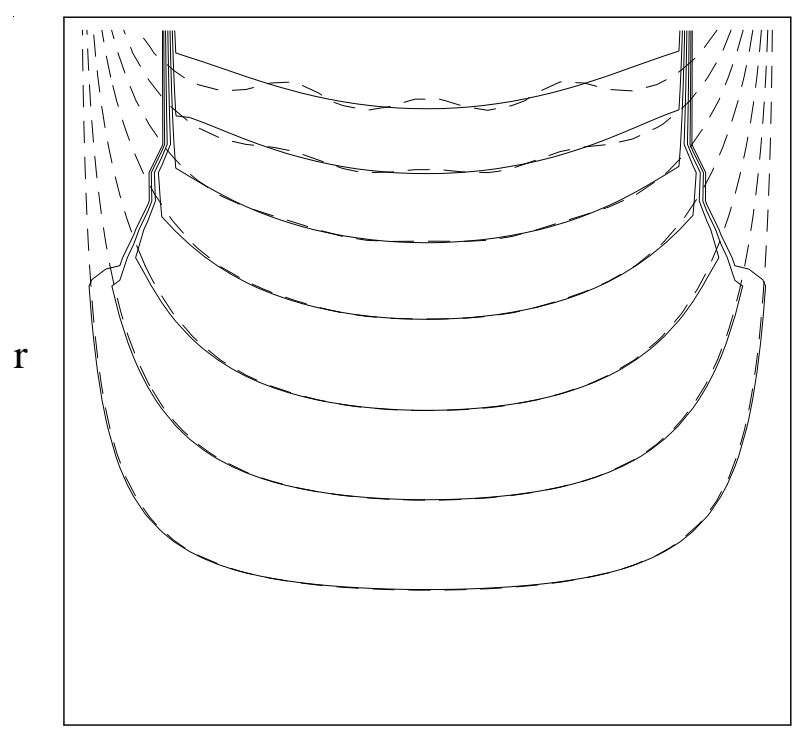

Z

Figure 1: Comparison of the analytical expanded field (dashed line) with SUPERFISH predicted field (solid line) at a maximum radius $0.24 \lambda$, maximum error is $6.7 \%$.

\section{AN APPLICATION TO BEAM DYNAMICS IN RF GUNS}

The field expansion off-axis derived in eq. 6 may be used to obtain higher order components in the transverse momentum $p_{r}$ imparted by the RF field to a photo-electron accelerated in a RF gun cavity. Assuming that the particle of charge $q$ and rest mass $m$ travels at $v=c$ on a trajectory parallel to the $\mathrm{z}$ axis, the transverse momentum $p_{r}$ at the cavity exit $\left(z=z_{f}\right)$, can be easily computed via the Panofsky-Wentzel theorem [3]:

$$
p_{r}=\frac{q}{m c}\left\{\left[A_{r}\left(z=z_{i}\right)-A_{r}\left(z=z_{f}\right)\right]+\int_{z_{i}}^{z_{f}} \nabla_{\perp} A_{z} d z\right\}
$$

Where the RF gun cavity starts with the metallic cathode wall at $z=z_{i}$, where the particles are generated. Since $A_{r}\left(z=z_{i}\right)=0$, and $A_{r}\left(z=z_{f}\right)=-E_{r} / \omega, p_{r}$ comes out to be not vanishing as in a standard multi-cell structure with open ends. Using the off-axis expansion of $\mathrm{TM}_{010-\pi}$ mode for the case $k=k^{\prime}$ of eq.6, we obtain:

$$
\begin{aligned}
p_{r}=\alpha \sin \phi & \left\{a_{1} k r+\sum_{n \geq 3} a_{n} \sum_{N=1}^{v} \frac{\left(n^{2}-1\right)^{N-1}}{4^{N} N !^{2}(N+1)}\left[\left(n^{2}-1\right)(k r)^{2 N+1}+\right.\right. \\
+ & \left.\left.4 N(N+1)(k r)^{2 N-1}\right]\right\}
\end{aligned}
$$

It is interesting to note that the first and third order components in eq.8, obtained setting $n=3$ and $N=1$, have been already evaluated in ref.[4],[5]

The interest of considering higher order terms in $p_{r}$ is relevant for all beam dynamics studies involving trajectories close to the cavity irises as in dark currents, beam halos, RF guns studies.

\section{DESIGN OF THE CAVITY}

Writing the family of the lines perpendicular to the equipotential lines of the field in the cavity, and setting initial conditions by specifying the iris aperture and radius of cavity equator, we obtain a system of parametric differential equation in $\zeta=\zeta(s)=k z$ and $\rho=\rho(s)=k r$ variables, written as follows:

$$
\left\{\begin{array}{c}
\frac{d \rho(s)}{d t}=\frac{\rho(s)}{Z_{0}} \sum_{n} a_{n} \cos (n \varsigma(s)) \sum_{N=0}^{v} \frac{\left(n^{2}-1\right)^{N}}{N !^{2}}\left(\frac{\rho(s)}{2}\right)^{2 N} \\
\frac{d \zeta(s)}{d t}=-\frac{\rho^{2}(s)}{2 Z_{0}} \sum_{n} n a_{n} \sin (n \varsigma(s)) \sum_{N=0}^{v} \frac{\left(n^{2}-1\right)^{N}}{N !^{2}(N+1)}\left(\frac{\rho(s)}{2}\right)^{2 N}
\end{array}\right.
$$

Whose solution gives the analytical approximation for the iris shown in fig.2, see [6]:

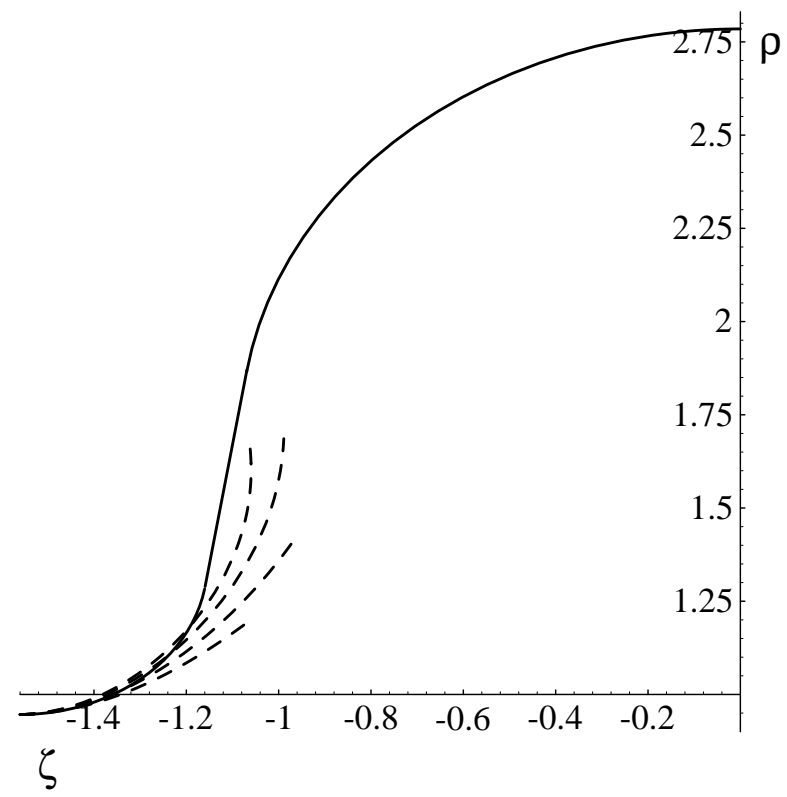

Figure 2: Comparison between actual cavity boundary (solid line) and analytical predictions (dashed lines) for different order of expansion $(\mathrm{N}=0,1,2,3)$.

\section{REFERENCES}

[1] S.Humpries "Principles of charged particle acceleration", John Wiley \& sons- New York, 1985

[2] P.M.Lapostolle, A.L.Septier "Linear accelerators", NorthHolland Publishing company - Amsterdam, July 1969 and S.C.Hartman, J.B.Rosenzweig, Phys.Rev.E-47 (1993) 2031 [3] W.K.H.Panofsky,W.A.Wenzel,Rev.Sci.Instr..27(1956)967

[4] L.Serafini, NIM A304(1991) 353-356, and ref. therein

[5] J.Gao, NIM A304(1991) 348-352

[6] D.Burrini, Grad. Thesis, University of Milan, Feb.1995 